\title{
LECTURES CRITIQUES
}

\section{Presses de Sciences Po | «Revue française de science politique »}

2019/5 Vol. 69 | pages 931 à 939

ISSN 0035-2950

ISBN 9782724636055

Article disponible en ligne à l'adresse :

https://www.cairn.info/revue-francaise-de-science-politique-2019-5-page-931.htm

Distribution électronique Cairn.info pour Presses de Sciences Po.

(C) Presses de Sciences Po. Tous droits réservés pour tous pays.

La reproduction ou représentation de cet article, notamment par photocopie, n'est autorisée que dans les limites des conditions générales d'utilisation du site ou, le cas échéant, des conditions générales de la licence souscrite par votre établissement. Toute autre reproduction ou représentation, en tout ou partie, sous quelque forme et de quelque manière que ce soit, est interdite sauf accord préalable et écrit de l'éditeur, en dehors des cas prévus par la législation en vigueur en France. Il est précisé que son stockage dans une base de données est également interdit. 


\section{CHRONIQUE}

BIBLIOGRAPHIQUE

VARIA

\section{LECTURES

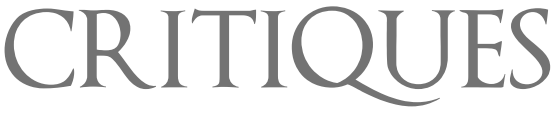

\section{_ La résistible ascension du populisme ${ }^{1}$ ?}

$\mathrm{L}$ 'ouvrage tout juste publié de Gilles Ivaldi est l'une des dernières livraisons du fleuve de récits, d'analyses, de plaidoyers, de réquisitoires sur le populisme. Alors que son émergence en Europe il $\mathrm{y}$ a un quart de siècle apparaissait comme un phénomène aux contours à la fois imprécis et limités dans le temps et dans l'espace, force est de constater que le populisme - nouveau pour l'Europe, plus ancien et enraciné de l'autre côté de l'Atlantique - fait désormais partie du paysage politique dans la quasi-totalité des démocraties, consolidées ou de fraîche création. Cette abondance de biens n'a pas pour autant dissipé toutes les interrogations et perplexités que cette innovation majeure dans le fonctionnement des partis et des systèmes politiques a suscité parmi les acteurs et observateurs médiatiques ou politologiques, à la recherche de similitudes ou différences au regard des catégories connues.

La tâche à laquelle s'attache G. Ivaldi est quelque peu herculéenne et le défi populiste qu'évoque le titre et qui sous-tend le "challenge" des nouveaux venus à l'encontre des valeurs, organisations et systèmes démocratiques peut aussi se lire comme un défi aux intellectuels et en premier lieu aux politologues dont on attend clarifications, éclairages et interprétations. Même si l'auteur est loin d'être le premier à se pencher sur la question, son objectif n'est pas inutile tant le consensus sur la «chose » est loin d'être établi. Et pour le faire, il ne lésine pas sur les moyens : puisque l'identification du populisme est si difficile, G. Ivaldi procède à un examen exhaustif dans le temps et dans l'espace, examine toutes ses manifestations et occurrences et, à partir de cet "échantillon", absorbe et inclut tout ce qui a pu être qualifié de «populisme » ou de "populiste » depuis la chute du Mur de Berlin. L'ambition n'est pas mince: elle nécessite de prendre en considération les dizaines ou centaines d'articles ou ouvrages qui se sont penchés sur tel ou tel aspect de la question, dans un ou plusieurs pays. À ma connaissance, l'auteur est le seul qui, au-delà de quelques ouvrages collectifs collaboratifs, ait tenté d'embrasser tout ou quasiment tout le champ quelque peu indéterminé du populisme contemporain. De ce point de vue, ce livre est et restera un instrument incomparable de connaissance et de réflexion sur l'objet. La bibliographie rassemblée et discutée est quasi exhaustive et sera précieuse pour quiconque voudrait s'inscrire dans la foulée et la prolonger. On peut toutefois regretter quelques lacunes, d'autant plus surprenantes dans une investigation qui embrasse si largement. À commencer par ce qui reste un classique précurseur demeurant d'une étonnante fraîcheur intellectuelle malgré le passage des années, Populism de Margaret Canovan (Harcourt Brace Jovanovich, 1980) dont n'est cité que l'article de 1999 dans Political Studies (« Trust The People! Populism and The Two Faces of The Democracy", $47[1])$. Ou encore les analyses de Pascal Perrineau sur le Front national dont ne sont évoquées que les études publiées en 2017. Ou enfin le silence total sur les publications de Marco Tarchi sur le populisme italien, pourtant incontournables sur la question dans la Péninsule. Malgré ces oublis

1. À propos de l'ouvrage de Gilles Ivaldi, De Le Pen à Trump. Le défi populiste, Bruxelles, Éditions de I'Université de Bruxelles, 2019 (UBlire), 404 p., bibliographie, annexes. 
régimes totalitaires. De là doit naître un type d'intellectuel inédit, dont la mission historique est d'ouvrir un nouvel espace des possibles politiques. Comme l'explique F. Dosse dans les dernières pages de son livre, l'ambition de cette saga « est de susciter le nécessaire travail de deuil des catégories de l'ancien monde et de faire place à une autoréflexion qui puisse, écartant les impasses du passé, jeter les bases d'un nouvel horizon d'attente et d'espérance, d'un avenir non tracé qui aurait retrouvé une boussole pour guider l'action de l'homme» (tome 2, p. 634).

Mais que faut-il entendre exactement par « intellectuel démocratique»? C'est d'abord un intellectuel qui reconnaît la validité du régime démocratique - avec tout le flou qui entoure un concept que G. Deleuze aurait pris plaisir à railler comme une «dent creuse». F. Dosse montre parfaitement comment les années 1980 ont été celles d'un réinvestissement de la démocratie : après le «moment Soljenitsyne ", après le génocide cambodgien, après les boat-people vietnamiens, les intellectuels font retour, à la manière de Claude Lefort, vers l'invention démocratique et les droits de l'homme. L'image des retrouvailles de J.-P. Sartre et R. Aron autour d'André Glucksmann, qui symbolise ce phénomène, est restée célèbre. Un intellectuel démocratique, ensuite, prend acte de la nécessité de soumettre ses arguments et ses positions à la discussion rationnelle : il ne s'agit plus de prononcer des discours en surplomb afin d'évangéliser Billancourt, mais d'entrer plus modestement dans l'espace public - aujourd'hui colonisé par les médias - afin d'y défendre une opinion éclairée qui ne se confond jamais avec la vérité nue. Comme l'explique F. Dosse en termes aristotéliciens, l'intellectuel doit dorénavant se contenter d'œuvrer, entre doxa et épistémè, dans le domaine de la doxazein, c'està-dire de l'opinion droite.

Dans une veine herméneutique, F. Dosse explique qu'il doit "jouer activement un rôle d'approfondissement démocratique grâce à son activité de veilleur dans les conflits d'interprétation» (tome 2, p. 631). Le rôle de ce "médiateur critique " est ainsi de contribuer à l'élaboration d'un «être-ensemble» qui soit «tourné vers la vie bonne dans des institutions justes " (tome 2, p. 631 et 634). De fait, c'est bien autour des questions du bien et du juste que les débats intellectuels se sont réorientés à partir des années 1980 en France, sous l'influence des développements de la théorie politique américaine de la décennie précédente $^{1}$. À cet égard, il est dommage que l'auteur interrompe son analyse en 1989 et ne se confronte pas aux évolutions de la configuration intellectuelle depuis cette date. Car il n'est pas certain que le chemin emprunté ait été celui qu'il appelle de ses vœux. L'éclatement du «front antitotalitaire " au début des années 2000, le retour de la critique sociale et l'apparition d'un néoconservatisme offensif semblent en effet indiquer que la civilisation des mœurs intellectuelles, qui s'est annoncée fugacement dans les années 1990, n'est plus vraiment à l'ordre du jour. Mais il est vrai que nul n'est prophète en son pays.

Gwendal Châton -

Université Rennes I, IDPSP

\section{Spectres de la violence ${ }^{2}$}

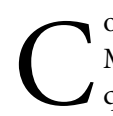
onnu pour ses travaux sur la violence au Moyen-Orient ou sur la question kurde, qu'il explore depuis plus de 20 ans, Hamit Bozarslan, directeur d'études à l'École des hautes études en sciences sociales (EHESS), articule cette fois sa réflexion sur le seul phénomène de la violence, aussi bien en Occident qu'en Orient. L'auteur s'étonne, à raison, que la violence demeure encore trop souvent un "angle mort" des réflexions sur le monde social, alors même que sa présence est continue, à basse ou haute intensité. Considérée comme épisodique, anormale, étrangère aux démocraties pacifiées, donc indigne d'intérêt, voire simplement effrayante, la violence ne génère qu'un faible investissement analytique chez les universitaires. Un déni parfois redoublé dans les chancelleries, qui ne semblent percevoir les contours de la violence que lorsqu'il est déjà trop tard. L'auteur indique ainsi que si les attentats dirigés contre les intérêts américains dans les années 1990 n'avaient pas été traités comme des faits divers isolés, la stratégie

1. Sur ce point, voir Mathieu Hauchecorne, La gauche américaine en France. La réception de John Rawls et des théories de la justice, Paris, CNRS Éditions, 2019. Ouvrage dont le lecteur trouvera une recension dans cette même chronique bibliographique.

2. À propos de l'ouvrage d'Hamit Bozarslan, Crise, violence et dé-civilisation. Essai sur les angles morts de la cité, Paris, CNRS Éditions, 2019, 480 p., bibliographie, index. 
d'Al-Qaïda conduisant au 11-Septembre aurait été parfaitement lisible. De fait, le terrorisme n'est pas une «menace» imaginaire, ou un simple leurre utilisé par les pouvoirs publics pour justifier de mesures sécuritaires, mais bien une violence délibérée contre les sociétés ${ }^{1}$.

Même s'il parait relever de "l'irraison", le choix de la violence par certains acteurs doit bien être pensé en tant que tel. Il s'arme sur des « circonstances", toujours jugées atténuantes pour ceux qui y recourent, sur des aspirations utopiques, sur une eschatologie religieuse ou séculière, sur un conspirationnisme généralisé, sur la glorification d'une pureté originelle ou sur une "nostalgie revivaliste" (p. 84). C'est visible dans l'abondante propagande écrite et visuelle de Daech, qui ne cesse de justifier ses actions, d'exalter la violence armée, le martyre et l'amour de la mort. La décision de recourir à la violence se fonde ainsi sur la volonté de préserver un ordre ou de le renverser à tout prix, d'anéantir un ennemi, sur un calcul rationnel quant à son efficacité et sur une projection de ses chances de réussite, même si l'issue est incertaine car ses modes d'action demeurent précaires. En témoigne l'effondrement du Sentier lumineux, pourtant assez structuré, au Pérou en 1992 après l'arrestation de son chef, Abimael Guzmán. Par ailleurs, si la violence est voulue, ses effets peuvent rapidement devenir anomiques. Ce fut le cas dans l'Algérie des années 1990, du côté du pouvoir comme de celui des islamistes, où la fuite en avant a donné naissance à une violence purement éradicatrice. Sous tous ces aspects, la violence politique n'apparaît plus comme relevant d'une quelconque culture des pays concernés ou comme un "legs de l'enfance sauvage de l'humanité " (p. 187), mais bien comme un principe et une solution exclusive jugée supérieure à toutes les autres.

Le choix de la violence se nourrit surtout de la «crise » entendue de façon large : crise politique, économique, de la conscience historique, du savoir et de l'autorité, qui frappe d'obsolescence les anciennes visions du monde. Devant l'incertitude et le changement sociétal, les sociétés musulmanes ont par exemple opté dès les années 1980 pour le conservatisme social et religieux, muselant leur société civile, bridant toute velléité d'individualisme libéral, tout en reconduisant un grand récit qui les verrait s'aliéner par l'occidentalisation et la colonisation. En Iran et en Irak, la guerre externe est devenue un outil de gestion de la crise interne et d'étouffement des revendications sociales, jusqu'à éteindre les " facultés cognitives de la société » (p. 154). Dans la Turquie de Receip Tayyip Erdogan, c'est au contraire une guerre interne interminable, aux ennemis variables, qui empêche les opposants ou les intellectuels de produire du sens. Partout d'ailleurs, le recours massif et stratégique à la désinformation, au mensonge et aux fausses nouvelles est devenu une violence contre l'esprit, qui désoriente les sociétés civiles et sape la confiance entre les groupes sociaux.

Face au même défi, le XXe siècle s'est abîmé en Occident dans la brutalisation des consciences et la violence guerrière qu'une approche en termes de lutte des classes échouerait à expliquer. Car d'autres divisions et passions étaient à l'œuvre : romantismes sombres, nationalismes bellicistes, utopies anti-universalistes, ou hiérarchies et assignations raciales, qui non seulement appelaient la violence, mais lui gardaient une place de choix après son utilisation, par fidélité à ceux qui avaient combattu et n'auraient pas compris qu'il faille $y$ renoncer. Si la crise peut manifester la désintégration d'un système, comme dans le cas de la France de 1789 , la guerre la pérennise, et son effet est imprévisible : la crise financière de 2008 a ainsi paradoxalement hâté la perspective d'une sortie du capitalisme, tout en portant au pouvoir, dans de nombreux pays, des dirigeants en phase avec le système capitaliste bancarisé, sécuritaires et protectionnistes, à commencer par Donald Trump aux États-Unis.

L'autonomisation de la violence, le choix de la violence pour la violence, à l'exception de toute solution politique, peut véritablement devenir « politicide» (p. 244). C'est-à-dire ne jamais créer les conditions pour que le projet qu'elle porte advienne. Ce seront les purges régulières contre les acteurs révolutionnaires, la refondation permanente par effacement du passé et, étonnamment, le désintérêt pour le régime politique mis en place, quand la délivrance n'est que religieuse et chiliastique. La mort de soi, dans l'attentatsuicide par exemple, devient secondaire, tant 
qu'elle terrorise la société et promet un salut éternel. Aussi bien dans le cas du nazisme que de l'État islamique, un processus nihiliste prend alors le pas sur la reconstruction politique annoncée, et la rationalité du recours à la violence devient délirante. Tout le projet politique dont la violence devrait accoucher est en fait souvent miné dès l'origine par un "désir de catastrophe » où les agents de la violence ne veulent pas seulement voir s'effondrer ce qui ne marcherait pas mais surtout ce qui marcherait mal, alors même qu'il y aurait suffisamment de routines et de régulations pour que le régime fonctionne. C'est ce que montrent la volonté du Royaume-Uni de quitter l'Union européenne (Brexit), la victoire de dirigeants autoritaires ou "illibéraux », et les phénomènes d'abstention électorale qui participent à «la production de la fatalité du pire par une forme démocratique d'abdication» (p. 406).

Ce qui intéresse plus profondément l'auteur, c'est la facilité avec laquelle la brutalisation des sociétés peut l'emporter historiquement sur leur "civilisation ", et la défaire. La civilisation chez Norbert Elias est caractérisée par H. Bozarslan de façon originale comme "manière d'habiter le monde ", dans l'assurance que la société est historiquement pacifiée par l'État, et que les échanges y sont confiants et durables. Les dispositifs coercitifs demeurent dans l'arsenal des démocraties mais, a priori, l'espace laissé aux revendications sociales et politiques vide de sens le recours à la violence des protestataires. C'est donc bien l'organisation du conflit qui préserve du conflit, tandis que son interdiction provoque la violence. Dès lors, le recours à la violence ne servira pas à fonder un ordre nouveau, mais à inverser les rapports de force. C'est ce qu'espèrent les mouvements indépendantistes et nationalistes, tels que l'Armée républicaine irlandaise (IRA) ou le Parti des travailleurs du Kurdistan (PKK), même si sur le temps long la discussion politique reprend ses droits et qu'ils sont obligés de renoncer à la lutte armée. Quant aux mouvements sans base sociale forte, comme la Fraction armée rouge (RAF) en Allemagne de l'Ouest, leur échec ouvre la voie à l'entrée de nouveaux groupes en politique, mais avec des demandes moins radicales.

Le phénomène singulier du $\mathrm{XX}^{e}$ siècle reste alors la transformation radicale de l'État en acteur de la destruction de la civilisation, et des sociétés dont il était le garant. Si le Léviathan hobbesien protège le groupe de sa puissance, le Béhémoth l'anéantit en portant la violence en son sein et non dans une guerre extérieure. Génocides, massacres, tortures et cruautés perpétrées par l'État même - comme récemment dans la Syrie de Bachar el-Assad - témoignent d'une sortie de la civilisation, et produisent en retour une défiance à l'égard d'un État qui a dévoilé sa propension à la férocité. De même, entre panarabisme, baasisme, talibanisme et posture à la R. T. Erdogan, le monde musulman est caractérisé depuis plusieurs décennies par une quête de puissance qui se fait au détriment d'un quelconque État protecteur ou démocratique, et introduit aussi des formes de brutalité dans les relations internationales. Cette violence a pu être au départ le fait de groupuscules, de marges politiques incultes - Hannah Arendt rappelait à quel point les chefs nazis étaient des "ratés »- ou d'une jeunesse revancharde, celle des Jeunes Turcs, justement, en 1908, mais aussi celle d'un Oussama Ben Laden, qui n'a que 23 ans lorsqu'il part combattre l'URSS en Afghanistan. La violence peut ensuite se déployer quand les groupes marginaux obtiennent une assise suffisante dans la population, ou fabriquent une unité organique, qui neutralise toute opposition et enfante cette "démocratie totalitaire » oxymorique dont rêvait Benito Mussolini. C'est bien la violence du régime nazi qui force N. Elias à amender son idée d'un processus de civilisation linéaire - et aussi, dit H. Bozarslan, à sortir d'un certain déni analytique - pour évoquer des "brèches dans la civilisation " et des moments de décivilisation, qui n'entament cependant pas la dynamique générale ${ }^{1}$. L'assise idéologique de tous ces mouvements est indéniable, comme l'est le millénarisme de l'islam politique, qui font prévaloir la seule volonté sur la réalité. "La dé-civilisation prend ainsi la forme d'un processus de sur-simplification par la violence, physique mais aussi cognitive, de ce que la civilisation avait complexifié ", écrit l'auteur (p. 375). Ainsi tombe la civilisation, dans la rencontre entre un besoin populaire de faire table rase et le triomphe d'acteurs sociaux violents, qu'ils appartiennent à l'appareil étatique ou le contestent.

Érudit, synthétique et placé sous le patronage critique d'Ibn Khaldûn, François Furet, ou encore

1. Norbert Elias, Les Allemands. Luttes de pouvoir et développement de I'habitus aux xIXe et XXe siècles, Paris, Seuil, 2017. 
Pierre Hassner, l'ouvrage de H. Bozarslan est enfin une réflexion sur la façon dont la prise en compte de la violence environnante peut conduire les universitaires à repenser la façon dont ils font des sciences sociales ${ }^{1}$. De Max Weber à H. Arendt, en passant par N. Elias encore, les guerres du $\mathrm{XX}^{e}$ siècle, et le phénomène totalitaire, vécus par ces auteurs dans leur chair, les ont amené à amender leur vision d'un État pacificateur - Léviathan devenu Béhémoth -, à proposer de nouveaux paradigmes et à repenser leur engagement dans la cité. "Étudier l'horreur, écrit H. Bozarslan, c'est déjà reconnaître, a posteriori, notre impuissance face à elle.» (p. 25) Travailler sur la violence revient alors à éveiller les consciences et à rappeler que les Lumières n'étaient pas équipées pour combattre les mythes vitalistes et biologiques du $\mathrm{Xx}^{\mathrm{e}}$ siècle, ou le culte de la personnalité des sociétés totalitaires. Pour sortir de la crise de la civilisation et des menaces que fait peser la brutalisation, il faut prendre acte de la fragilité intrinsèque des démocraties et donner comme identité politique aux individus un "vouloir-vivre-démocratique ", une citoyenneté humaniste et rationnelle. Revendiquant, en conclusion, une posture normative, l'ambition théorique du livre est de penser un État qui ne soit plus seulement une instance hobbesienne de coercition et de domination mais serait limité dans sa volonté de puissance afin d'organiser au mieux le dissensus; sans perdre de vue qu'il doit produire suffisamment de puissance pour se défaire de ses marges violentes et assurer une cité démocratique universelle.

Emmanuel Taïeb Sciences Po Lyon, Triangle

1. La taille de l'ouvrage a visiblement compliqué le travail éditorial : «Nietzsche » est mal orthographié cinq fois et «Henry Rousso » trois fois, notamment dans l'index nominatif. 